\title{
Experimental Digital Video Recording Using Co-Cr-Ta/Ni-Fe Double-layered Tape \\ (NHK)
}

\section{Structure and characteristics of the Co-Cr-Ta/Ni-Fe tape}

\begin{tabular}{lc}
\multicolumn{2}{c}{ Deposition conditions of $\mathrm{Co}-\mathrm{Cr}-\mathrm{Ta} / \mathrm{Ni}-\mathrm{Fe}$ film } \\
\hline Composition & \\
Co-Cr-Ta & $\mathrm{Co}_{79.5} \mathrm{Cr}_{18} \mathrm{Ta}_{2.5}$ at. $\%$ \\
Ni-Fe & $\mathrm{Ni}_{80} \mathrm{Fe}_{20}$ at. $\%$ \\
Sputtering gas & $\mathrm{Kr}$ \\
Gas pressure & $1 \mathrm{mTorr}$ \\
Temperature of base film & $135^{\circ} \mathrm{C}$ \\
\hline \hline
\end{tabular}

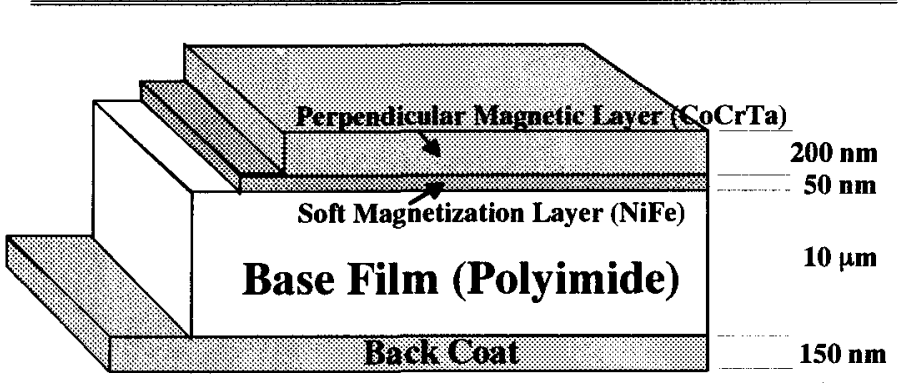

Magnetic properties of $\mathrm{Co}-\mathrm{Cr}$-Ta film

\begin{tabular}{lrl}
\hline Ms & 460 & emu/cc \\
$\mathrm{Hc}_{\perp}$ & 1250 & $\mathrm{Oe}$ \\
\hline
\end{tabular}

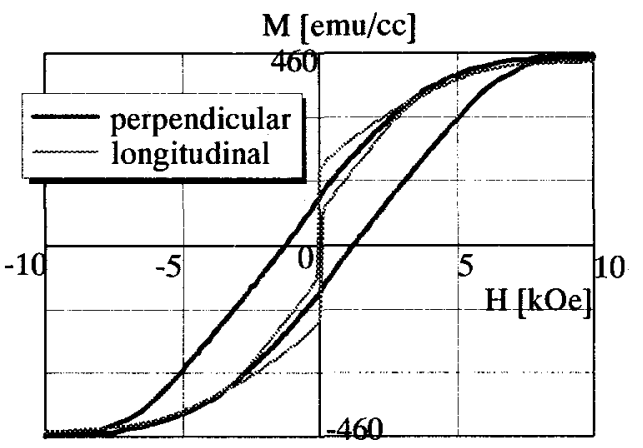

Magnetization Loops of the $\mathrm{Co}-\mathrm{Cr}$-Ta/NiFe Film

Structure of Tape

\section{Read/Wite characteristics of the Co-Cr-Ta/Ni-Fe tape}

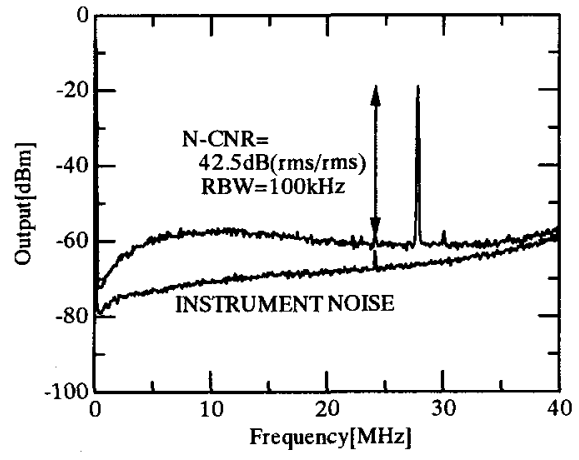

Reproduced signal spectrum and instrument noise spectrum

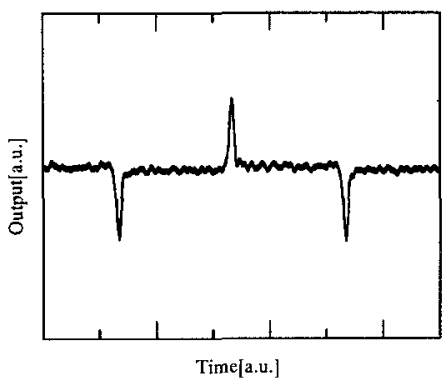

Reproduced wave form after phase equalization
Specifications of the experimental VCR

\begin{tabular}{c|c}
\hline Drum diameter & $40 \mathrm{~mm}$ \\
Drum rotation speed & $90 \mathrm{rps}(5400 \mathrm{rpm})$ \\
Relative tape-to-head velocity & $11.4 \mathrm{~m} / \mathrm{s}$ \\
Total recording bit rate & $126 \mathrm{Mbps}$ \\
Maximum recording frequency & $27.7 \mathrm{MHz}$ \\
Number of recording channels & 2 \\
Channel coding & $8-14 \mathrm{modulation}$ \\
Number of tracks per field & 6 \\
Shortest recording bit length & $0.18 \mu \mathrm{m}$ \\
Head type & $\mathrm{Ring}$ \\
Head track width & $5 \mu \mathrm{m}$ \\
\hline
\end{tabular}

Read/Write characteristics

\begin{tabular}{c|c}
\hline \hline $\begin{array}{c}\text { CN ratio ( RBW=100kHz ) } \\
\text { Byte error rate }\end{array}$ & $\begin{array}{c}42.5 \mathrm{~dB}(\mathrm{rms} / \mathrm{rms}) \\
1 \times 10^{-5}\end{array}$ \\
\hline \hline
\end{tabular}

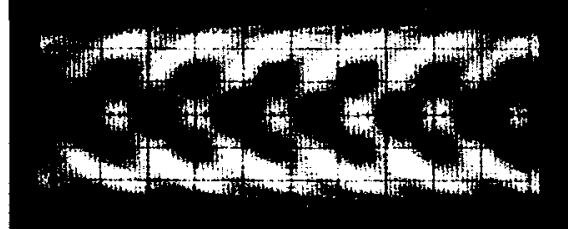

Eye pattern of reproduced signal 